\title{
Predictors of long-term survival in patients treated with targeted temperature management after cardiac arrest
}

\author{
Jiři Bonaventura ${ }^{1}$, David Alan ${ }^{1}$, Jiři Vejvoda ${ }^{1}$, Markéta Pavlíkováª , Josef Veselka ${ }^{1}$
}

\begin{abstract}
$1^{1}{ }^{\text {nd }}$ Medical School, Charles University, Motol University Hospital, Prague, Czech Republic 2Department of Probability and Mathematical Statistics, Faculty of Mathematics and Physics, Charles University, Prague, Czech Republic
\end{abstract}

Submitted: 2 November 2017

Accepted: 1 January 2018

Arch Med Sci 2020; 16 (5): 1250-1253

DOI: https://doi.org/10.5114/aoms.2019.81397

Copyright $\odot 2019$ Termedia \& Banach

Sudden cardiac arrest (CA) is a common cause of death in industrialized countries. The incidence of out-of-hospital cardiac arrest (OHCA) is estimated at 350,000 to 700,000 patients in Europe every year [1]. The survival rate of OHCA used to be very poor, but advances in cardiopulmonary resuscitation and post-cardiac arrest care including targeted temperature management (TTM) have improved outcomes in selected cohorts of patients [2]. Neurologic injury from cerebral hypoxia is the most common cause of death in patients with OHCA [3]. While the issue of OHCA is well described and the data are widely available, there is little evidence regarding TTM after in-hospital cardiac arrest (IHCA), and our knowledge is based mostly on the results of retrospective and observational studies $[4,5]$. Whether certain subpopulations of cardiac arrest patients have greater benefit from TTM is being debated [6]. The aim of this study was to identify the independent predictors of long-term neurologically favourable survival in patients treated with TTM after CA.

The study was performed on 140 consecutive patients (29 women, mean age: $64.6 \pm 12.8$ years), who were hospitalized between November 2006 and June 2016 in a single cardiovascular centre after successfully resuscitated CA. Both patients with OHCA and IHCA were included. All patients had return of spontaneous circulation (ROSC) for at least $20 \mathrm{~min}$ and were unconscious (a score of $<8$ on the Glasgow Coma Scale) on admission to the hospital. All patients underwent TTM as soon as possible after admission. All patients with suspected acute coronary syndrome underwent cardiac catheterization as soon as possible, while the patients with ST-segment elevation myocardial infarction underwent emergent cardiac catheterisation with immediate percutaneous coronary intervention, when required. No emergency or urgent cardiovascular surgery was performed. An intra-aortic balloon pump (IABP) was used in 16 patients. Extracorporeal membrane oxygenation (ECMO) was used in 2 patients. All patients who survived to hospital discharge underwent regular clinical and telephonic follow-up.

The primary endpoint was long-term neurologically favourable survival. Good neurologic function was defined as a Cerebral Performance Category (CPC) of 1 or 2. Bad outcome was defined as CPC 3 to 5 . A total of 136 patients were included in the statistical analysis. Four patients (all men) were lost to follow-up. Baseline characteristics of patients and cardiac arrest features are shown in Table I. In the entire cohort of patients,

\author{
Corresponding author: \\ Jiři Bonaventura MD \\ $2^{\text {nd }}$ Medical School \\ Charles University \\ Motol University \\ Hospital \\ V Úvalu 84 \\ 15006 Prague \\ Czech Republic \\ Phone: +42 0224434901 \\ E-mail: jiri.bonaventura@ \\ fnmotol.cz
}


Table I. Cardiac arrest characteristics $(n=136)$

\begin{tabular}{|c|c|}
\hline Parameter & Value, $n(\%)$ \\
\hline \multicolumn{2}{|l|}{ Aetiology: } \\
\hline STEMI & $49(36)$ \\
\hline NSTEMI & $11(8)$ \\
\hline Multivessel heart disease & $23(17)$ \\
\hline Chronic heart failure & $17(13)$ \\
\hline Pulmonary embolism & $4(3)$ \\
\hline Primary arrhythmia & $17(13)$ \\
\hline Respiratory & $12(9)$ \\
\hline Sepsis & $2(1)$ \\
\hline Other & $1(1)$ \\
\hline \multicolumn{2}{|l|}{ First monitored rhythm: } \\
\hline Ventricular fibrillation & $70(51)$ \\
\hline Non-perfusing ventricular tachycardia & $8(6)$ \\
\hline Asystole & $21(15)$ \\
\hline Pulseless electrical activity & $2(1)$ \\
\hline Other & $17(12)$ \\
\hline Unknown first rhythm & $18(13)$ \\
\hline \multicolumn{2}{|l|}{ Location of cardiac arrest: } \\
\hline $\mathrm{OHCA}$ & $110(81)$ \\
\hline $\mathrm{IHCA}$ & $26(19)$ \\
\hline
\end{tabular}

STEMI - ST-segment elevation myocardial infarction, NSTEMI myocardial infarction without ST-segment elevation, OHCA - outof-hospital cardiac arrest, IHCA - in-hospital cardiac arrest.

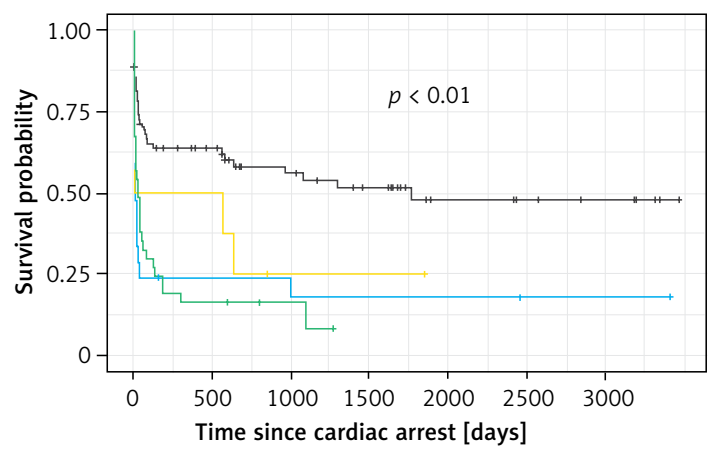

Initial rhythm $+\mathrm{VF}+\mathrm{VT}+$ Asystole

Numbers of patients at risk

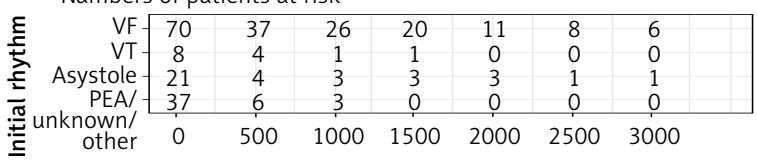

Figure 1. Kaplan-Meier estimator of the probability of neurologically favourable survival after cardiac arrest - by initial rhythm. Groups with a low count of events were joined together to improve performance of statistical testing and graphic clarity

$V F-$ ventricular fibrillation, VT - ventricular tachycardia, PEA - pulseless electrical activity. the incidence of primary endpoint was $34.6 \%$. The mean follow-up was $4.3 \pm 2.8$ years, maximum 9.6 years. There were statistically significant differences in the primary endpoint among groups of patients with different initial rhythm (log-rank test $p<0.01$, Figure 1). Patients who had ventricular fibrillation (VF) as the initial rhythm had the most favourable survival (52.9\%). There were statistically significant differences in the primary endpoint among groups of patients with different CA causes (log-rank test $p=0.03$, Figure 2). $100 \%$ annual mortality was observed among the patients whose CA was caused by pulmonary embolism $(n=4)$ or chronic heart failure $(n=17)$, as shown in Figure 2 . All patients with documented typical symptoms and signs of heart failure and corresponding structural and/or functional cardiac abnormality were included in the heart failure group. The mean left ventricle ejection fraction was $28.6 \pm 7.9 \%$. Multivariate analysis was performed to identify the joint effect of independent predictors of longterm neurologically favourable survival. We identified three statistically significant predictors of neurologically favourable survival: i) younger age (1 year of age increases the risk of a bad outcome by $\mathrm{HR}=1.05,95 \% \mathrm{Cl}: 1.03-1.07 ; p<0.01)$, ii) ventricular fibrillation as the initial rhythm (decreased

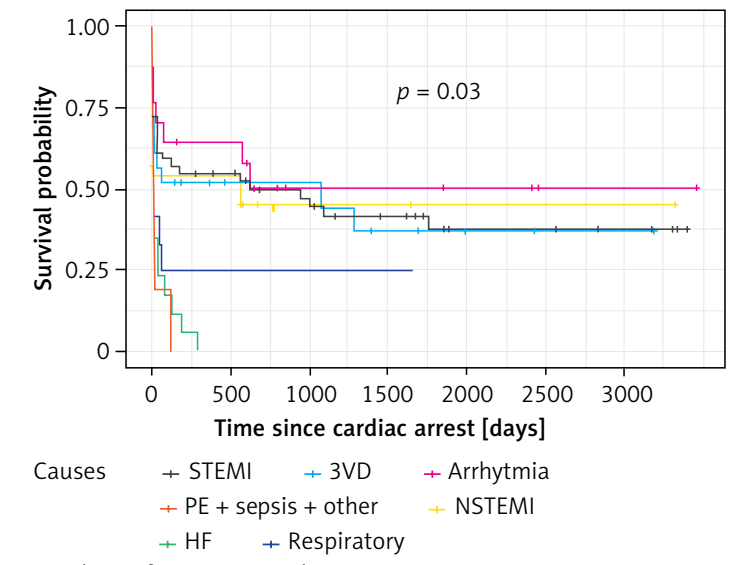

Numbers of patients at risk

\begin{tabular}{|c|c|c|c|c|c|c|c|}
\hline STEMI & 49 & 24 & 17 & 13 & 7 & 6 & 4 \\
\hline NSTEMI & 11 & 6 & 2 & 2 & 1 & 1 & 1 \\
\hline $3 \mathrm{VD}$ & 23 & 8 & 8 & 4 & 3 & 1 & 1 \\
\hline $\mathrm{HF}$ & 17 & 0 & 0 & 0 & 0 & 0 & 0 \\
\hline Arrhytmia & 17 & 10 & 4 & 4 & 3 & 1 & 1 \\
\hline Respiratory & 12 & 3 & 2 & 1 & 0 & 0 & 0 \\
\hline $\mathrm{PE}+$ sepsis + & 7 & 0 & 0 & 0 & 0 & 0 & 0 \\
\hline & 0 & 500 & 1000 & 1500 & 2000 & 2500 & 3000 \\
\hline
\end{tabular}

Figure 2. Kaplan-Meier estimator of the probability of neurologically favourable survival after cardiac arrest - by cause of cardiac arrest. Groups with a low count of events were joined together to improve performance of statistical testing and graphic clarity

STEMI - ST-segment elevation myocardial infarction, NSTEMI - myocardial infarction without ST-segment elevation, 3VD - three-vessel coronary artery disease, $H F$ - chronic heart failure, PE - pulmonary embolism. 
chance of bad outcome by $\mathrm{HR}=0.40,95 \% \mathrm{Cl}$ : $0.25-0.63 ; p<0.01$ ), iii) primary arrhythmia (without structural cardiac disease) as an aetiology of cardiac arrest (decreased chance of bad outcome by $\mathrm{HR}=0.43,95 \% \mathrm{Cl}: 0.21-0.91 ; p=0.03)$. The Kaplan-Meier survival curves according to the initial rhythm and aetiology are shown in Figures 1 and 2 .

There are many subpopulations of CA patients, and their prognosis varies greatly. At present, there is sufficient evidence that TTM improves the outcome after adult-witnessed OHCA caused by ventricular fibrillation (VF) [7]. Our study results correspond with this knowledge, identifying the initial ventricular fibrillation as an independent predictor of good long-term prognosis.

Cardiac arrest mostly occurs in patients with known or previously unrecognized heart disease. However, it can occur in persons without any identifiable structural abnormality. Patients with ventricular arrhythmia require an evaluation to determine whether structural heart disease is present. In our study, those patients were put together in the group of "primary arrhythmia" aetiology, if normal findings on ECG, echocardiography and cardiac catheterization were present at the initial evaluation. The majority of patients without apparent structural heart disease likely do not actually have "normal" hearts, but our diagnostic tools limit identification of structural or functional abnormalities. Currently, several aetiologies of CA are recognized with clearly defined electrophysiological abnormalities, including Wolff-Parkinson-White syndrome, long QT syndrome, the Brugada syndrome or catecholamine-induced polymorphic ventricular tachyarrhythmia [8]. In the remainder, no such abnormalities are identifiable. Such patients are categorized as having idiopathic ventricular tachycardia. Among our study cohort, a total of 17 patients were included in the "primary arrhythmia" aetiology of CA. None of the above electrophysiological abnormalities were diagnosed in any patient. However, in the subsequent examination in the follow-up (e.g. magnetic resonance imaging or endomyocardial biopsy), 4 of those patients were diagnosed with cardiomyopathy: i) arrhythmogenic right ventricular cardiomyopathy, ii) left ventricular non-compaction cardiomyopathy, iii) hypertrophic cardiomyopathy, iv) amyloidosis. For statistical purposes, we decided to leave them in the primary group, since the cardiomyopathy was not known at the initial evaluation.

An extremely poor prognosis was observed among the patients after CA caused by chronic heart failure. All patients $(n=17)$ after CA caused by chronic heart failure died within the first year after CA, irrespective of heart failure aetiology. Ischaemic aetiology was described in 13 patients, non-ischaemic aetiology in 4 patients. Two of them had severe aortic stenosis, while the other two had severe non-ischaemic mitral regurgitation. None of them underwent surgical or percutaneous intervention of their valvular heart disease. There are several mechanisms of death in patients with chronic heart failure. The most common are: i) sudden cardiac death caused by ventricular arrhythmia and ii) pump failure as the opposite extreme. In patients with moderate to severe chronic heart failure, overall mortality shows a progressive increase, more often as a consequence of disease progression, whereas the relative proportion of sudden deaths decreases [9, 10]. Only 3 out of 17 patients had shockable rhythm on their initial ECG. It is very likely that in our cohort of patients, the disease progression of heart failure was the main cause of CA. Therefore, their prognosis was determined at the very beginning, irrespective of our efforts in post-cardiac arrest care including TTM. The bad outcome of these patients could have been improved by using ECMO. Nevertheless, the mean age of all patients after CA caused by chronic heart failure was $69 \pm 8.9$ years, so more conservative treatment was chosen.

Our study has several limitations. First, it is a retrospective and non-randomized study performed in a single centre. Second, due to the retrospective design of the study, we were missing some data about the details of CA. Data provided by emergency services are often incomplete and cannot be obtained by further investigation, e.g. the initial rhythm, time from collapse to cardiopulmonary resuscitation or the exact time to ROSC. Third, we acknowledge that our results might not be applicable to all CA patients since our cohort included mainly cardiac causes of CA, as shown in Table I. Fourth, the analysis of patients after CA caused by pulmonary embolism or chronic heart failure is of limited value due to the low included numbers and the sparse clinical data at hand. We also cannot be certain that these data are entirely representative and generalizable for non-tertiary referral centres with less experience in the management of patients after CA.

In conclusion, the independent predictors of long-term neurologically favourable survival after successfully resuscitated CA treated with TTM are: lower age, primary arrhythmia as an aetiology of cardiac arrest and ventricular fibrillation as an initial rhythm. Patients after CA caused by pulmonary embolism or chronic heart failure have a poor prognosis.

\section{Conflict of interest}

The authors declare no conflict of interest. 


\section{References}

1. Sasson C, Rogers MA, Dahl J, Kellermann AL. Predictors of survival from out-of-hospital cardiac arrest a systematic review and meta-analysis. Circ Cardiovasc Qual Outcomes 2010; 3: 63-81.

2. Nolan JP, Ferrando P, Soar J, et al. Increasing survival after admission to UK critical care units following cardiopulmonary resuscitation. Crit Care 2016; 20: 219.

3. Nolan JP, Neumar RW, Adrie C, et al. Post-cardiac arrest syndrome: epidemiology, pathophysiology, treatment, and prognostication. Resuscitation 2008; 79: 350-79.

4. Nichol G, Huszti E, Kim F, et al. Does induction of hypothermia improve outcomes after in-hospital cardiac arrest? Resuscitation 2013; 84: 620-5.

5. Chan PS, Berg RA, Tang Y, et al. Association between therapeutic hypothermia and survival after in-hospital cardiac arrest. JAMA 2016; 316: 1375-82.

6. Bonaventura J, Alan D, Vejvoda J, Honek J, Veselka J. History and current use of mild therapeutic hypothermia after cardiac arrest. Arch Med Sci 2016; 12: 1135-41.

7. Nolan JP, Soar J, Cariou A, et al. European Resuscitation Council and European Society of Intensive Care Medicine Guidelines for Post-resuscitation Care 2015. Resuscitation 2015; 95: 202-22

8. Hayashi M, Shimizu W, Albert CM. The Spectrum of epidemiology underlying sudden cardiac death. Circ Res 2015; 116: 1887-906.

9. Pocock SJ, Ariti CA, McMurray JJ, et al. Predicting survival in heart failure: a risk score based on 39372 patients from 30 studies. Eur Heart J 2013; 34: 1404-13.

10. Rahimi K, Bennett D, Conrad N, et al. Risk prediction in patients with heart failure. JACC Heart Fail 2014; 2: 440-6. 